\title{
A Study on the Effect of Gaussian Noise on PSNR Value for Digital Images
}

\author{
Parminder Kaur and Jagroop Singh
}

\begin{abstract}
Most of the rules relating simple nonlinear threshold values for wavelet-based de-noising have assumptions that the wavelet coefficients are independent values. However, when we talk of natural images, we observe that wavelet coefficients have significant dependency. The phrase Peak Signal to Noise Ratio, often abbreviated PSNR, is an [9] engineering term for the ratio between the maximum possible power of a signal and the power of corrupted noise that affects the fidelity of its representation. In this paper, experimentation is performed to study the effect of Increasing Gaussian noise on PSNR and the corresponding measure. The last section of the paper illustrates the above explained concept with the graphical view of PSNR with increase in noise.
\end{abstract}

Index Terms - PSNR, Image, Wavelet, Gaussian Noise, MRE.

\section{INTRODUCTION}

There are various methods to remove noise from the image and hence one can increase Peak Signal To Noise (PSNR) ratio. Some prominent work proposed by [1] as implement de-noising method by using the dual-tree complex wavelets into the ordinary ridgelet transform. The paper [1] used shift invariant property of the dual-tree complex wavelet and the high directional sensitivity of the ridgelet transform. In that paper, digital complex ridgelet transform is applied for de-noising of some standard images embedded in white noise Here, it used hard thresholding of the complex ridgelet. Experimental results show that the new method is better than VisuShrink, the ordinary ridgelet image denoising. The paper [1] used wiener filter that is available in the Matlab Image Processing Toolbox. Complex ridgelets could be applied to curvelet image de-noising as well.

In paper, [2] a better method is described for removing additive white noise of known variance from photographic images. The technique is based on a characterization of statistical properties of natural images represented in complex wavelet decomposition. Specially, the paper decompose the noisy image into wavelet sub-bands or patterns, estimate the autocorrelation of both the noise-free raw coefficients and their magnitudes (within each sub-band or pattern), impose these statistics by projecting onto the space of images having the desired autocorrelations. It reconstructs an image from the modified wavelet coefficients. De-noising results compare favorably to three reference

Manuscript received February 28, 2011.

Parminder Kaur is M.Tech- Student at Deptt. Of ECE, DAVIET, Jalandhar, Punjab, India.

Jagroop Singh is working as faculty in Deptt. Of ECE, DAVIET, Jalandhar, Punjab, India. methods, both perceptually and in terms of mean squared error.

The paper, [3] discusses a method for additive random noise that can easily be removed using simple threshold methods. De-noising of natural images corrupted by Gaussian noise using wavelet techniques is very effective because of its ability to capture the energy of a signal in few energy transform values. In [3], wavelet de-noising scheme thresholds the wavelet coefficients arising from the standard discrete wavelet transform. In [3], it is proposed to investigate the suitability of different wavelet bases and the size of different neighborhood on the performance of image de-noising algorithms in terms of PSNR.

The paper [4], proposed regional characteristics of the images are associated with Information clusters. It gives novel approach of information theory based Minimum Relative Entropy (MRE) and Entropy methods for image compression. In this paper, a two stage compression process is performed through homogenous MRE method, and heterogeneous MRE. The compressed images are reconstructed through Region Growing technique. The performance of image compression and restoration is analyzed by the estimation of parametric values such as Mean Square Error (MSE) and Peak Signal to Noise Ratio (PSNR). According to [4], higher the PSNR better the reconstruction (as shown in figure1) process. In [4], Six radiographic medical images of various sizes are analyzed and Maximum PSNR of 33 is achieved.

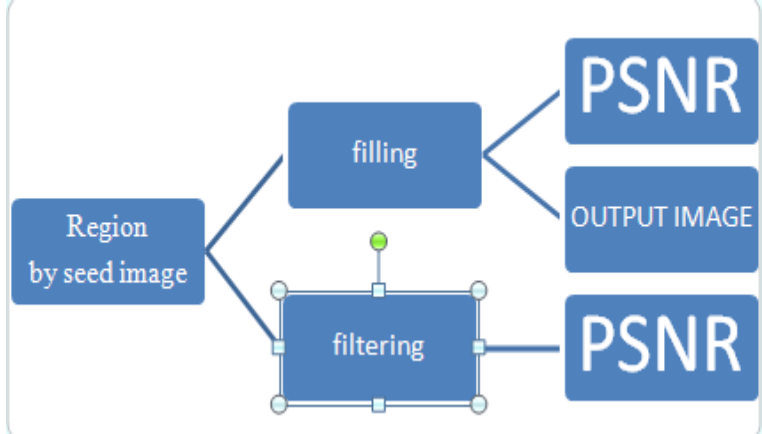

Figure 1. Reconstruction through region growing

The paper [5] describes a method for removing noise from digital images, based on a statistical model of the coefficients of an over complete multi-scale oriented basis. In this paper, Neighborhoods of coefficients at adjacent positions and scales are modeled as the product of two independent random variables: a Gaussian vector and a hidden positive scalar multiplier. The latter modulates the local variance of the coefficients in the neighborhood, and is thus able to account for the empirically observed correlation between the coefficient amplitudes. In [5], Bayesian least squares 
estimate of each coefficient reduces to a weighted average of the local linear estimate over all possible values of the hidden multiplier variable. It demonstrates with the images contaminated by additive white Gaussian noise that the performance of the proposed method substantially better in terms of mean squared error.

The paper [6] proposes new heavy-tailed bivariate PDF (Prior Density Function) to model the statistics of wavelet coefficients, and a simple nonlinear threshold function (shrinkage function) is derived from the PDF using Bayesian estimation theory. In the paper, the new shrinkage function does not assume the independence of wavelet coefficients.

The paper [7] describes an approach to image de-noising which is done by utilizing the inter-scale dependency of wavelet coefficients. It analyzes a new property of parent children type statistics in the wavelet domain. Then, a Gaussian mixture model (GMM) is employed to fit this statistical property, where the children's variance field is estimated by a linear relation involving their parent. Lastly, MMSE estimates for the noisy wavelet coefficients are obtained. The paper compares proposed approach with other competing approaches. In particular, though the proposed approach does not outperform other approaches at all noise levels, there are occasions when it does outperform other approaches and where it may fall short of outperforming other approaches, the difference in performance is not large.

The paper [8] proposes a new method for image de-noising based on the contourlet transform, which has been recently introduced. Image de-noising by means of the contourlet transform introduces many visual artifacts due to the Gibbs-like phenomena. Due to the lack of translation invariance of the contourlet transform, [8] employs a cycle-spinning-based technique to develop translation invariant contourlet de-noising scheme. This scheme achieves enhanced estimation results for images that are corrupted with additive Gaussian noise over a wide range of noise variance. The experiments in [8] show that the proposed approach outperforms the translation invariant wavelets both visually and in terms of the PSNR values, especially for the images that include mostly fine textures and contours.

\section{NOISE AND PSNR}

The phrase Peak Signal to Noise Ratio, often abbreviated PSNR, is an [9] engineering term for the ratio between the maximum possible power of a signal and the power of corrupted noise that affects the fidelity of its representation. As many signals have wide dynamic. The MSE and PSNR values are defined as shown in eq. (1):

$$
\begin{gathered}
\text { MSE }=\frac{1}{m n} \sum_{i=0}^{m-1} \sum_{j=0}^{n-1}\|I(i, j)-K(i, j)\|^{2} \\
P S N R=10 \log _{10}\left(\frac{M A X_{I}^{2}}{M S E}\right)=20 \log _{10}\left(\frac{M A X_{I}}{\sqrt{M S E}}\right)
\end{gathered}
$$

Here, $\mathrm{MAX}_{\mathrm{I}}$ is the maximum pixel value of the image. When the pixels are represented using 8 bits per sample, this is 255. Here, $I$ and $K$ are two $m \times n$ monochrome images where one of the images is considered a noisy approximation of the other.

Compression systems like JPEG include optional pre-processing with filtering to avoid compression artefacts. At higher compression ratios a stronger filtering is needed that impacts the large scale [10] image content. Here, typical diffusion processes is applied before the blockwise DCT compression using the peak signal to noise ratio (PSNR) as an objective quality measure. We give a simple measure of artefact reduction in terms of PSNR, and show that a considerable artefact reduction is achieved by pre-processing at the same bit rate as and with no greater error than the original compression.
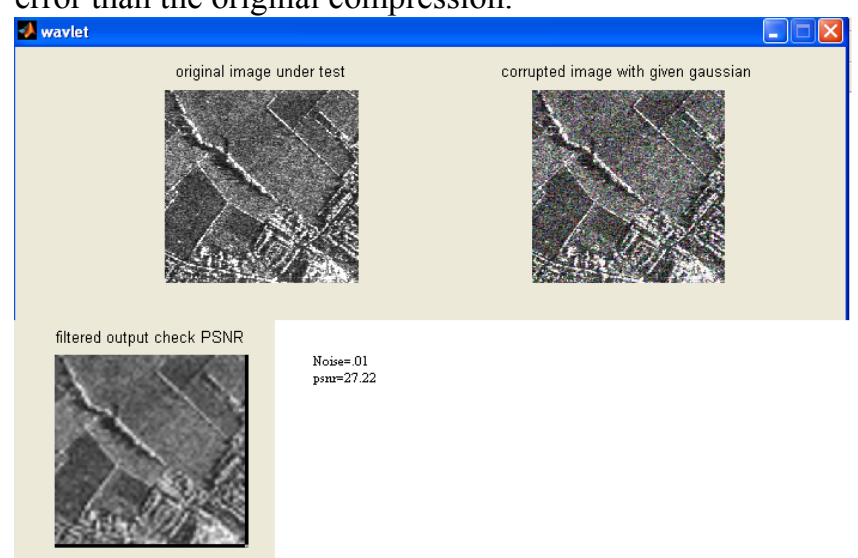

Figure 2. NOISE $=.01, \mathrm{PSNR}=27.22$

The artefact reduction implies a better subjective impression of quality. The images processed with the PSNR-based algorithm had nearly the same but greater PSNR value as the original compression and lesser Gaussian noise. Subjects preferred noisy image content to the lack of small scale details, so the subjective preference of the images with reduced artefact is worse than that of the original compression. Results suggest, however, that non-linear diffusion is more efficient for artefact reduction than non-adaptive smoothing as given Gaussian filtering in terms of the subjective preference.

In the figure 2, the graphical variation of noise with PSNR value is given. As the content of Gaussian noise is low better is the PSNR in this case Noise is 0.01 and the value of PSNR is 27.22 .

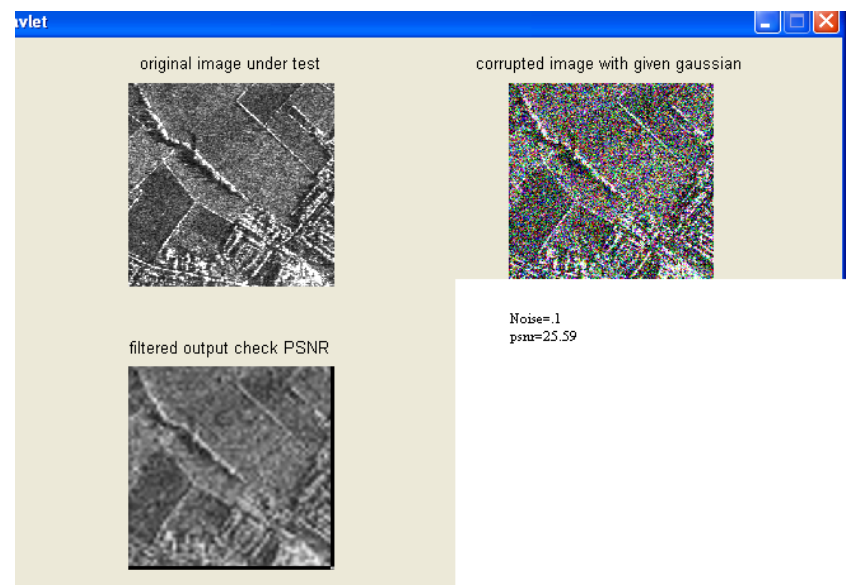

Figure 3. NOISE $=.1, \mathrm{PSNR}=25.59$

In the figure, the graphical variation of noise with PSNR value is given for Gaussian noise of .1 (one tenth of previous) and the value of PSNR is 25.59. 


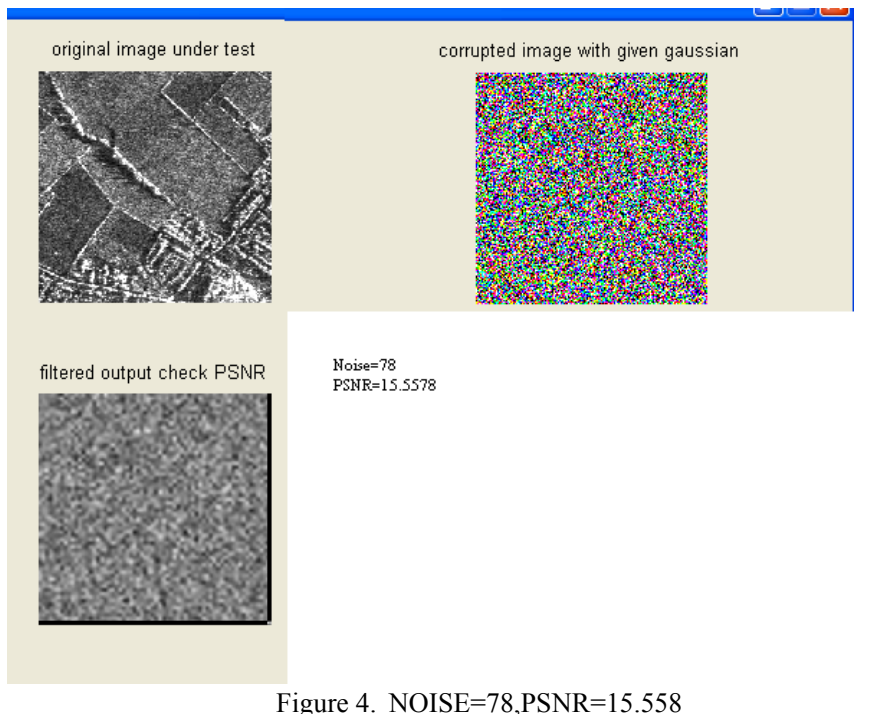

In the figure 4, the graphical variation of noise with PSNR value is given for Gaussian noise of 78 and the value of PSNR is reduced very much that is 15.558 .

\section{CONCLUSION AND GRAPHICAL OUTPUT}

Lossy image and video compression yield typical error patterns on the decompressed images or video sequences due to the quantization error. The paper [10] explains that depending on the compression scheme and the bit rate, these can be ringing patterns around the edges, false or blurred texture, visible block-boundaries in block-partitioning schemes. These phenomena are called compression artefacts. Compression artefacts not only deteriorate the visual quality of images but also perturb image processing and machine vision algorithms like edge detection and motion estimation. A possible solution is to do a post-processing on the decompressed image, which removes compression artefacts. Some post-processing methods improve the visual quality of the compressed image by various adaptive filters that alleviate the artefacts and enhance the edges. The blocking artefact for the blockwise transform compression can also be reduced by prediction methods for the quantized or missing transform coefficients. Post-processing can also be formulated as a reconstruction problem for the original image and solved by optimization with respect to an objective error measure like MSE, to probability considerations, to regularity constraints, or to a combination of these. The quality of the compressed images is affected not only by the bit rate and the compression algorithm, but also by the data source. In particular, images with more details usually degrade more than those with fewer details when compressed at the same bit rate. It is therefore sensible to do some pre-processing on the image before compression, which alleviates the components of the image susceptible to artefacts. We define what we mean by Noise reduction, and use a simple way of measuring and expressing it through PSNR. To check whether Noise reduction results in images that are more preferred by human observers, subjective tests were done to rate the different methods. Results on test images suggest that the block boundaries and false textures are alleviated in the pre-processed images. The diffusion strength is controlled by PSNR measurements. The graphical view suggest that as we increase the noise or Gaussian content to the test image more prominent is the distortion and hence lesser is the value of PSNR.

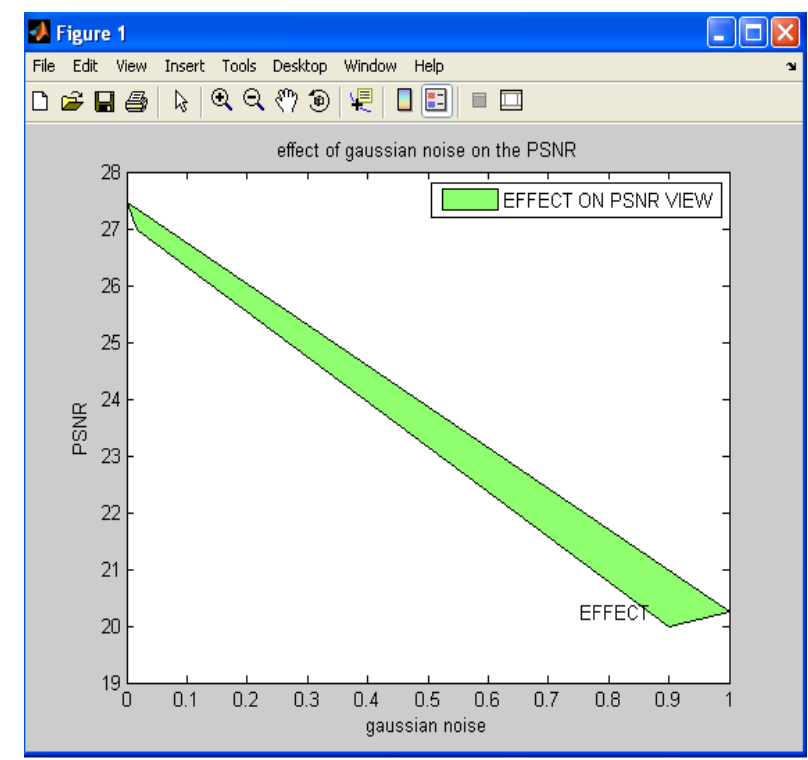

Figure 5. NOISE $=\mathrm{X}-\mathrm{Axis}(0-1), \mathrm{PSNR}=\mathrm{Y}-\mathrm{Axis}(19-28)$

As shown in figure 5, $\mathrm{X}$-axis shows the range for Gaussian noise from $0-1$ in our case and corresponding PSNR from the $\mathrm{y}$-axis keep on decreasing with the increment in the noise.

From the figure 5, we can calculate any sudden impact of the noise on the PSNR and hence, calculationare easy to understand.

\section{REFERENCES}

[1] G. Y. Chen and B. Kegl , "Complex Ridgelets for Image Denoising", Pattern Recognition, Vol. 40, 2007, pp. $578-585$.

[2] Javier Portilla and Eero P. Simoncelli, "Image Denoising Via Adjustment Of Wavelet Coefficient Magnitude Correlation", IEEE Computer Society Proceedings of the 7th ICIP, 2000, pp. 10-13.

[3] S. Kother Mohideen,. S. Arumuga Perumal, M. Mohamed Sathik, "Image De-noising using Discrete Wavelet transform", IJCSNS International Journal of Computer Science and Network Security, Vol. 8, No. 1, January 2008, pp. 213-216.

[4] R. Sukanesh, R. Harikumar, N.S.Balaji and S. R. Balasubramaniam, "Analysis of Image Compression by Minimum Relative Entropy (MRE) and Restoration through Weighted Region Growing Techniques for Medical Images", Engineering Letters, 14:1, EL 14.1.16 (Advance online publication: 12 February 2007)

[5] Javier Portilla, Vasily Strela, Martin J. Wainwright, Eero P. Simoncelli, "Image Denoising using Scale Mixtures of Gaussians in the Wavelet Domain", IEEE Transactions on Image Processing, vol. 12, no. 11 pp. 1338-1351, November 2003.

[6] Levent S, endur, Ivan W. Selesnick, "A Bivariate Shrinkage Function For Wavelet-Based Denoising", IEEE Transactions On Signal Processing, Vol. 50, No. 11, November 2002.

[7] P. Chen and D. Suter, "A Simple Pixel-Adaptive Bayesian Approach to Image Denoising Using Wavelet Interscale Dependency", Technical Report MECSE-1-2002, Monash University, Clayton 3800, Australia.

[8] Ramin Eslami, Hayder Radha, "Translation-Invariant Contourlet Transform and Its Application to Image Denoising", IEEE Transactions on Image Processing, 15(11), pp. 3362-3374, 2006.

[9] O. Young, "Synthetic structure of industrial plastics", In Plastics, 2nd ed. vol. 3, J. Peters, Ed. New York: McGraw-Hill, 1964, pp. 15-64.

[10] Shahriar Kaisar, Md.Sakib Rijwan, Jubayer Al Mahmud, Muhammad Mizanur Rahman, "Salt and Pepper Noise Detection and removal by Tolerance based Selective Arithmetic Mean Filtering Technique for image restoration", IJCSNS International Journal of Computer Science and Network Security, VOL.8 No.6, June 2008271

[11] Ivan Kopilovic, Tamás Szirányi, "Comparing Objective and Subjective Quality Results for Compression Pre-processing with Non-linear Diffusion",. Proceeding Scale Space'03 Proceedings of the 4th international conference on Scale space methods in computer vision, Springer-Verlag Berlin, Heidelberg, 2003, pp. 729-743. 